\title{
Effects of MK-801 on acquisition, retention, and cross-modal transfer of active avoidance behavior in rats
}

\author{
EUGENE R. DELAY \\ Regis University, Denver, Colorado
}

\begin{abstract}
Three experiments were conducted to examine the effects of MK-801, a noncompetitive NMDA antagonist, on acquisition, retention, and cross-modality transfer (CMT) of learning of an active avoidance response in rats. The first showed that MK-801 $(0.1 \mathrm{mg} / \mathrm{kg})$ disrupted acquisition but not retention of avoidance responding when an auditory intensity cue signaled shock. In the second experiment, the effects of MK-801 on CMT were examined by training rats to avoid shock with a visual intensity cue in the first session, then with an auditory intensity cue in two more sessions. Different groups of rats were given MK- 801 (either 0.05 or $0.1 \mathrm{mg} / \mathrm{kg}$ ) prior to one of the three sessions. When MK-801 was injected before visual training, the drug blocked acquisition of the avoidance task, preventing the formation of memory usable during the transfer session. When MK-801 was given before the second (transfer) session, rats did not show evidence of transfer from the previous training with the visual cue, nor did they show evidence of new learning. In the third experiment, MK- 801 had no effect on avoidance behavior when it was administered immediately after training. These data suggest that NMDA receptors are involved not only in simple discrimination learning but also in neuronal processes responsible for transfer of learning between sensory modalities.
\end{abstract}

For over a decade there has been growing interest in the functional significance of $N$-methyl-D-aspartate (NMDA) receptors in certain types of cognitive processes. Numerous reports suggest that NMDA receptors have a role in working memory, spatial memory, and related learning and memory functions. Dizocilpine maleate or (+)-MK-801 hydrogen maleate (MK-801) is a noncompetitive NMDA receptor antagonist that has been shown to impair acquisition of olfactory discriminations (G. S. Robinson, Crooks, Shinkman, \& Gallagher, 1989), passive avoidance (Parada-Turska \& Turski, 1990; Venable \& Kelly, 1990), spatial tasks such as the Morris water maze (Heale \& Harley, 1990) and radial-arm maze (Caramanos \& Shapiro, 1994; Shapiro \& O'Connor, 1992), and classical conditioning (Cox, Guthrie, Macrae, \& Kehoe, 1994; G. B. Robinson, 1993). Although in numerous reports it has appeared that MK-801 does not affect recall, in a few it has been found that MK-801 can either impair (Lee, Lee, Wang, \& Lin, 1993) or improve retention (Mondadori, Weiskrantz, Buerki, Petschke, \& Fagg, 1989; Weiskrantz \& Mondadori, 1991) of specific types of passive avoidance responses.

Little is known, however, about the role of NMDA receptors or the effects of MK-801 on transfer of learning between tasks, including those involving transfer between sensory modalities. Cross-modality transfer (CMT)

This research was supported in part by a Faculty Development Grant from Regis University. Correspondence concerning this article should be addressed to E. R. Delay, Department of Psychology, Regis University, 3333 Regis Blvd., Denver, CO 80221 (e-mail: psych.regis. edu). can be demonstrated when a subject learns a task with cues in one modality and then learns the task again but with cues in a second modality. Such a subject is able to learn the second task at a faster rate than a subject without the prior experience of the first modality (Church \& Meck, 1983). Typically, CMT has a positive transfer effect that is more potent if there is a relational similarity underlying the information that is transferred-for example, if both cues are increases in stimulus intensity. In the only study (Davis \& Klinger, 1994) to test the effects of MK-801 on CMT, goldfish were conditioned to suppress branchial responses when either light or tone cues were paired with shock. Transfer testing in nondrug fish showed CMT from the tone to the light cue but not from light to the tone. MK-801 administered prior to the first session could block acquisition to either cue. However, if the goldfish were first trained with the light cue and then given MK-801 prior to transfer testing with the tone cue, the previous training with the light cue prevented MK801 from blocking acquisition to the tone. This is particularly important since in nondrug goldfish CMT was undetectable for this training sequence, suggesting that the facilitatory effects of CMT can override the detrimental effects of MK-80l during acquisition. It is not known, however, whether CMT interacts with the effects of MK801 in a similar fashion in mammalian species.

In this study, three experiments were conducted to examine the effects of MK- 801 on acquisition, retention, and CMT of an active avoidance response in rat subjects. In the first, the effects of MK-801 on the acquisition and retention of active avoidance responding were examined when the onset of shock was cued by an increase in in- 
tensity of an auditory stimulus. In the second, the effects of MK-801 on CMT were examined by initially training the avoidance response with a visual intensity cue, then training the response with the auditory intensity stimulus. In the third experiment, the effects of MK-801 on consolidation processes were examined by administering the drug immediately after training.

\section{EXPERIMENT 1}

Most of the previous research on the effects of MK801 on avoidance behavior employed a variety of passive avoidance procedures. Consequently, the first experiment was conducted to determine whether MK-801 would alter acquisition or retention of an auditory intensity avoidance response in a four-way shuttle device by administering the drug to a different group of rats before one of four training sessions. Locomotion and escape latencies also were monitored to see whether drug-induced changes in activity would influence active avoidance responding.

\section{Method}

Subjects. Thirty-two male albino Sprague-Dawley rats between 90 and 100 days of age were housed individually in the home colony. Food and water were available ad lib. Colony lighting was maintained on a 12:12-h light:dark cycle, with the lights turned on at 8:00 a.m. Each rat was tested at the same time each day during the light portion of the cycle.

Apparatus. The shuttle apparatus measured $60 \mathrm{~cm}$ square $X$ $57 \mathrm{~cm}$ high and was painted uniformly white. The grid floor was divided into four equal-size quadrants by 5 - $\mathrm{cm}$-high partitions. Each quadrant of the floor, composed of 0.6-cm-diameter metal rods spaced $1.27 \mathrm{~cm}$ apart, could be individually electrified with scrambled shock. During each session, an incandescent red light mounted above one corner of the shuttle apparatus provided $5 \mathrm{~lx}$ of background light. Pseudorandom white noise stimuli were generated by an SN76477 integrated circuit (Texas Instruments) coupled to an amplifying circuit (Delay, Golden, \& Steiner, 1978) and presented through two speakers mounted in opposite walls of the shuttle. Noise intensity between trials measured $60 \mathrm{~dB}$ (re: $20 \mu \mathrm{N} / \mathrm{m}^{2}$, A scale), and at the onset of a conditioned stimulus (CS), noise intensity increased to $70 \mathrm{~dB}$. The rise and fall times of the CS followed a negatively accelerating function, reaching $85 \%$ of full intensity change within $100 \mathrm{msec}$ and full change within the next $50 \mathrm{msec}$.

Procedure. Each rat was handled for $5 \mathrm{~min}$ and then allowed to explore the shuttle apparatus for $10 \mathrm{~min}$ on 6 consecutive days before the first session. Each rat was given 30 shock avoidance trials on each of 4 consecutive days. Shock intensity $(0.2-$ $0.3 \mathrm{~mA}$ ), determined for each subject on the last day of habituation, was set just above the threshold value that elicited locomotion but below an intensity that would produce a ceiling effect on avoidance responding. During a trial, the onset of the CS was followed $5 \mathrm{sec}$ later by the onset of the unconditioned stimulus (US). The rat could avoid or escape shock and terminate the CS by entering any other quadrant. Intertrial intervals were random and ranged between 20 and $40 \sec (M=30 \mathrm{sec})$. To check for changes in response to shock during a session, response latencies were measured for one noncued escape trial before and one after each session. Also, since the highest dose tested $(0.1 \mathrm{mg} / \mathrm{kg})$ can increase activity (Whishaw \& Auer, 1989), the number of avoidance responses could be artificially elevated by an increase in spontaneous movement from one quadrant to another. Therefore, the number of quadrants entered by each rat during the session was recorded. At the end of a training session, the apparatus was cleaned with a weak ammonia solution, rinsed, and dried.

Prior to the experiment, each rat was randomly assigned to one of four drug conditions ( $n=8$ /group). In this and subsequent experiments, each drug condition is identified with a sequence of $S$ (saline) and M (MK-801) letters, which indicates the order in which saline and MK-801 were administered. Rats assigned to the first condition, designated as MSSS, received intraperitoneal injections of $0.1-\mathrm{mg} / \mathrm{kg} \mathrm{MK}-801((5 \mathrm{R}, 10 \mathrm{~S})-(+)-5-$ methyl-10,11dihydro-5H-dibenzo[a,d]cyclohepten-5,10-imine hydrogen maleate, obtained from Research Biochemicals International) mixed in a $0.1-\mathrm{mg} / \mathrm{ml}$ solution before the first session and an equivalent volume of saline vehicle $(0.9 \% \mathrm{NaCl})$ solution before each of the other three sessions. The second group (SMSS) received MK-801 prior to the second session, the third group (SSMS) received the drug prior to the third session, and the fourth group (SSSM) was given the drug prior to the fourth session. Each group received saline before the remaining sessions. Each rat was given the appropriate injection in the colony room $40 \mathrm{~min}$ before the first trial of each session and then placed in the shuttle apparatus $10 \mathrm{~min}$ before the first trial.

\section{Results}

In general, the rats reached asymptotic levels of avoidance responding by the second session, unless they were given MK- 801 before the first training session. Rats given MK-801 prior to the first session did not show any improvement in performance until the third session. The drug did not alter avoidance behavior of other groups, and it did not produce a detectable change in escape latencies or locomotor activity in any group.

The number of avoidance responses emitted during the 30 trials of each session was analyzed with analysis of variance procedures treating drug conditions (4) as a between-subjects variable and the sessions (4) as a within-subjects variable. This analysis indicated a significant main effect of sessions $[F(3,84)=93.40, p<$ $.001]$ and a significant interaction between drug conditions and sessions $[F(9,84)=5.05, p<.001$; see Figure 1$]$. Since drug effects, if any, would occur as part of the interaction, the data were partitioned by session and by drug condition and then subjected to analysis of variance procedures and to Newman-Keuls post hoc comparisons where appropriate (Howell, 1987).

The analyses comparing avoidance responding of the groups within each session did not reveal group differences except in the second session $[F(3,28)=9.165, p<$ $.001]$, when the rats assigned to the MSSS condition emitted significantly fewer avoidance responses than did any other group $(p<.01)$. All four analyses examining the performance of rats assigned to each drug condition across sessions showed significant changes in responding of every group [all $\left.F_{\mathrm{s}}(3,28) \geq 11.4, p<.001\right]$. Consequently, each set of data was examined with NewmanKeuls tests $(p<.01)$. For the MSSS group, avoidance responding did not change between Sessions 1 and 2, but then increased significantly in Session 3 to a level equivalent to that seen in Session 4 (see Figure 1). By contrast, rats in each of the other drug conditions showed a significant increase in avoidance responding from Session 1 to a level in Session 2 that was equivalent to avoidance responding in Sessions 3 and 4. 


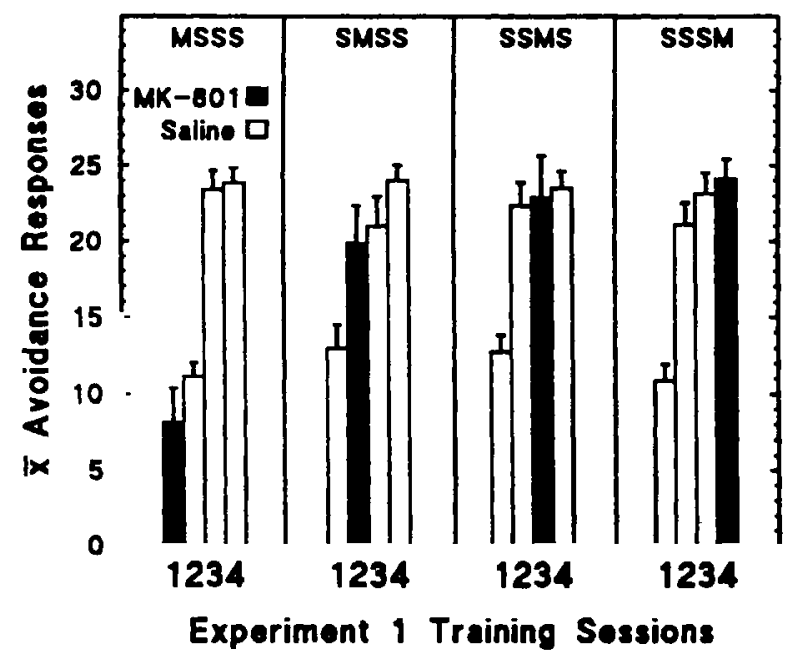

Figure 1. The mean number $( \pm S E M)$ of avoidance responses emitted by the rats in each of the four groups in each training session with a noise intensity CS. The letters identifying each group indicate whether the group received $0.1-\mathrm{mg} / \mathrm{kg} \mathrm{MK-801}$ (M) or saline (S) before each of the four sessions.

The possibility of drug-induced sensory and motor effects that might affect avoidance responding was examined by analyzing the escape latency and activity measures. For the escape latencies, an analysis of variance for mixed designs was used to detect differences between groups (4), between the pre- and postsession escape trials (2), and across sessions (4). No significant main or interactive effects of MK-801 on latencies were found in this experiment (all $F \mathrm{~S}<1.0$; MK-801 $M=4.39 \mathrm{sec}$, $S E M= \pm 0.56$; Saline $M=4.40 \mathrm{sec}, S E M= \pm 0.26$ ). To check for a potential elevation in avoidance responding due to increased activity, a "false alarm" response rate for each session was computed by dividing the number of times a rat moved from one quadrant to another by the duration of the session. Neither measure included the trial or the $5 \mathrm{sec}$ immediately following the end of the trial. An analysis of variance comparing false alarms for the four groups across the four sessions did not detect any systematic changes (all $F_{\mathrm{S}}<1.0$; MK- $801 M=1.56$, $S E M= \pm 0.17$; Saline $M=1.41, S E M= \pm 0.08$ ).

\section{Discussion}

Rats receiving saline before the first session reached asymptotic levels of avoidance responding on the 2 nd day. This indicates that most of the learning occurred during the first session, unless the rats were given MK801. While the MSSS rats appeared able to perform the avoidance task, the lack of improvement in avoidance responding between the first and second session suggests that MK-801 administered before the first session interfered with formation of long-term memory of the avoidance task. The absence of drug effects on performance of rats in the other drug conditions indicate that MK-801 did not have an effect on memory retrieval. In addition, the lack of drug effects on escape latencies and false alarm responding suggests that the drug did not change avoidance responding by altering the rats' responses to shock or their activity.

\section{EXPERIMENT 2}

The first experiment showed that MK-801 impairs acquisition but not retention or retrieval of the active avoidance task when an auditory intensity cue is used. The second experiment was conducted to examine the effects of MK-801 on CMT of learning with the avoidance task. Previous studies with this task (Delay, 1986, 1988) have consistently shown that CMT between the visual and auditory modalities is most potent when the cues in the two modalities are relationally and dimensionally similar - for example, when both cues involve an increase in stimulus intensity. Therefore, the visual and auditory intensity cues that produced the greatest amount of CMT in the earlier experiments were used to maximize the potential effects of CMT in this experiment. Finally, different groups were given one of two doses of MK-801 before one of three training sessions to assess the effects that the drug might have on processes related to CMT.

\section{Method}

Subjects. The subjects were 64 male albino Sprague-Dawley rats $90-100$ days of age maintained in the same manner as described for Experiment 1.

Apparatus. The shuttle apparatus and the equipment used to generate the noise stimuli were the same as in the first experiment. For visual avoidance training, the red light was replaced by two incandescent lights positioned side by side and centered over the shuttle apparatus. A 20-W light provided 50-lx background illumination. CS illumination, with rise and fall times similar to those of the noise cue, was $270 \mathrm{~lx}$, produced by a $100-\mathrm{W}$ light. In addition, the auditory stimuli were replaced with masking noise ( $48 \mathrm{~dB})$ from a room ceiling fan.

Procedure. Habituation and training procedures were identical to those of the first experiment with the following exceptions. During the 1st day of habituation, the rats were exposed to the red light and 60- $\mathrm{dB}$ white noise conditions described in Experiment 1. On the $2 n d$ day, they were habituated to $50-1 \mathrm{x}$ white incandescent light and the room fan. These conditions were alternated daily for 8 days prior to the first training session.

Sixteen rats were randomly assigned to each of four drug conditions. Rats in three of the four conditions were trained with the visual $\mathrm{CS}$ in the first training session and with the noise CS in two subsequent sessions. One group (MSS) was given MK-801 $40 \mathrm{~min}$ before the first training session, one group (SMS) was given MK801 before the second session, and one group (SSM) was given the drug before the third session. Half of the rats in each drug condition were given $0.05 \mathrm{mg} / \mathrm{kg}(n=8)$ and the other half were given $0.1 \mathrm{mg} / \mathrm{kg}$. Both doses were mixed in equal-volume solutions. All rats received saline before the other two sessions. The fourth drug condition was a saline control condition (SSS). These rats received saline $40 \mathrm{~min}$ before all three sessions. Eight of the 16 rats in the SSS group were trained with the visual CS during the first session and with the auditory CS during the next two sessions. The rest of the rats were trained with the noise cue in all three sessions. 


\section{Results}

First, the data for the rats tested in the SSS conditions were examined with a two-factor analysis of variance for mixed designs and Newman-Keuls tests to characterize the nature of CMT in the present experiment. Then, to compare the SSS control rats with rats tested with the two doses in the three MK-801 drug conditions, avoidance data were examined with a two-factor analysis of variance treating the eight groups as a between-subjects variable and the three sessions as a within-subjects variable. These data were then partitioned by session and by drug condition for further analysis.

SSS conditions. The results of the SSS conditions indicated that the rats trained with the visual CS during the first session exhibited positive transfer of learning to the auditory CS (see Figure 2). An analysis of variance comparing the performance of the two SSS groups over the three sessions showed a significant increase in the number of avoidance responses over sessions for these rats $[F(2,28)=49.56, p<.001]$ but no differences between groups were detected. Newman-Keuls testing showed $(p<.05)$ that both groups exhibited CMT with significantly higher levels of avoidance responding in the second and third sessions than seen in the first session. Similar effects of CMT were seen in the scores of the first two sessions for the rats in the SSM condition $[F(2,28)=$ $99.12, p<.001]$.

MK-801 conditions. As can be seen in Figure 2, in general MK-801 blocked acquisition of the avoidance response with the light cue (MSS group) in the first session. When MK-801 was administered before the second

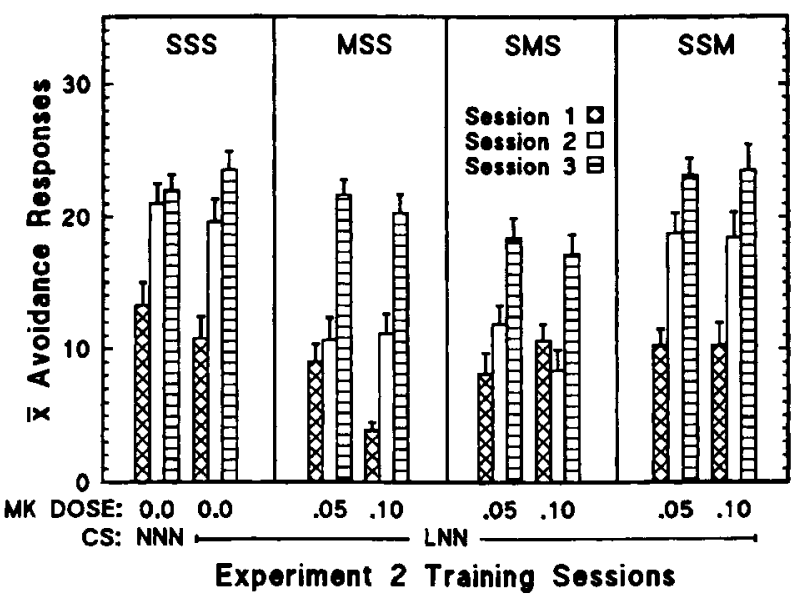

Figure 2. The mean number $( \pm S E M)$ of avoidance responses emitted by the rats in each of the eight groups in each of the three training sessions. The letters identifying each group indicate whether the group received MK-801 (M) or saline (S) before each of the three sessions. Except for the SSS condition, one group in each drug sequence received $0.05-\mathrm{mk} / \mathrm{kg} \mathrm{MK-801}$ and the second group received $0.1-\mathrm{mg} / \mathrm{kg}$. Seven groups were trained with a light (L) intensity $C S$ in the first session and a noise $(N)$ intensity $C S$ in the second and third sessions (cross-modal transfer). The eighth group was trained with the noise $\mathrm{CS}$ in all three sessions. session (SMS group), the drug disrupted the positive transfer effects of CMT from the light cue and blocked acquisition with the auditory cue. No effect of the drug was seen when the drug was administered before the third session (SSM group). The analysis of the avoidance data yielded significant differences for the main effects of groups $[F(7,56)=6.07, p<.001]$ and sessions $[F(2,112)$ $=242.72, p<.001]$ and for the interaction between these two variables $[F(14,112)=4.68, p<.001$; see Figure 2]. To detect any drug effects that were part of the interaction, in subsequent analyses these data were partitioned by sessions and by drug conditions.

Significant group differences in avoidance responding were seen in each session. In the first session, the significant group effect $[F(7,56)=3.46, p<.005]$, according to Newman-Keuls comparisons $(p<.05)$, was the result of significantly fewer avoidance responses by the MSS group given $0.1 \mathrm{mg} / \mathrm{kg}$ than by all other groups. This suggests that the higher dose of the drug may have interfered with the ability of the MSS rats to perform the task. The analysis of the avoidance data for the second session also revealed significant group differences $[F(7,56)=$ $9.44, p<.001]$. Post hoc testing showed that scores of the rats in the MSS and the SMS conditions were similar to each other, but all of these groups emitted significantly fewer avoidance responses than rats tested in the SSS or the SSM conditions. No differences due to dose in the second session were detected, however. The analysis of the data for the third session also indicated a significant group effect $[F(7,56)=2.76, p<.025]$. Interestingly, the SMS group given $0.1-\mathrm{mk} / \mathrm{kg}$ MK-801 emitted significantly fewer avoidance responses than did the rats in the SSM or the SSS conditions. Similarly, the SMS group given $0.05 \mathrm{mk} / \mathrm{kg}$ emitted significantly fewer avoidance responses than did any of these groups, except for the SSS group given auditory training every day. A separate analysis showed that avoidance responding of the SMS groups during the third session did not differ from responding of the rats in the SSS and the SSM conditions $(F<1.0)$ in the second session. Collectively, these data suggest that in the SMS group, MK-801 interfered with processes associated with transfer of learning and new acquisition.

To detect any other effects related to dose within a drug sequence, the performance of the two groups tested under each drug condition were compared over the three sessions. Only the analysis of the MSS groups showed a significant dose-related effect $[F(2,28)=3.52, p<.05]$ and that was due to the small number of avoidance responses emitted in the first session by the group given $0.1-\mathrm{mg} / \mathrm{kg} \mathrm{MK}-801$ (see Figure 2).

The analysis of false alarm data revealed a small but significant increase $(p<.025)$ in the false alarm rate related to MK- $801(M=2.41 / \mathrm{min}, S E M= \pm 0.16)$ as compared with the rate observed with saline $(M=2.04 / \mathrm{min}$, $S E M= \pm 0.08$ ). No group differences in false alarm rates were detècted, however. Escape latencies were unaffected by MK-801 (all $F_{\mathrm{S}}<1.0$ ). 


\section{Discussion}

Previous studies reported transfer of learning between visual and auditory modalities with this avoidance task (Delay, 1986, 1988). Like those found in earlier studies, the effects of CMT in this study were seen primarily in the second session in the avoidance performance of the SSS rats and the SSM rats. In the second session, rats initially trained with the visual CS emitted avoidance responses to the new auditory $\mathrm{CS}$ at a level nearly equivalent to that seen in rats initially trained with the auditory CS. However, in the MSS condition, MK-801 appeared to block the transfer effects from the visual training, probably by preventing learning from occurring during the first session. Curiously, avoidance responding of the rats in the SMS condition did not increase during the second session after receiving MK-801, indicating that MK-801 prevented the SMS rats from utilizing previously learned information that involved a different sensory modality. Moreover, their responding in the third session appeared to be equivalent to that seen in the second session of the SSS control groups and the SSM groups, suggesting that the drug impaired new learning when these rats were trained with a new cue, even though elements of the task (e.g., the CS-US temporal relationship, responses) were familiar. On the other hand, the performance of the rats in the SSM condition indicated that, as in the first experiment, MK-801 did not affect recall of the auditory discrimination once it was learned.

In general, both doses of MK-801 were equally effective in altering avoidance responding - with the exception that the higher dose may have interfered with the ability of the MSS rats to perform the avoidance response. However, none of the other groups responded as if the drug hampered their ability to execute the avoidance response.

\section{EXPERIMENT 3}

In the first two experiments, $\mathrm{MK}-801$ could have affected processes related to memory formation and CMT that occur after the training session as well as during. Therefore, a third experiment was conducted to examine the effects of MK-801 administered immediately after the training session.

\section{Method}

Subjects. Twenty-eight male albino rats (Sprague-Dawley) between 90 and 100 days of age were housed and maintained as described above.

Apparatus. The apparatus described for Experiment 2 was used in this experiment.

Procedure. The procedures used in previous experiments were modified as follows. The rats were randomly assigned to four groups ( $n=7 /$ group) prior to the first of three training sessions, one each day for 3 consecutive days. Two groups were trained with the noise intensity CS in all three sessions. One group (MSS) received $0.1-\mathrm{mg} / \mathrm{kg}$ MK-801 (IP) in the home colony within $1 \mathrm{~min}$ after the end of the first training session, and the second group (SMS) received the drug after the second training session. Saline was administered on the opposite day. The other two groups were trained with the visual intensity CS during the first session and

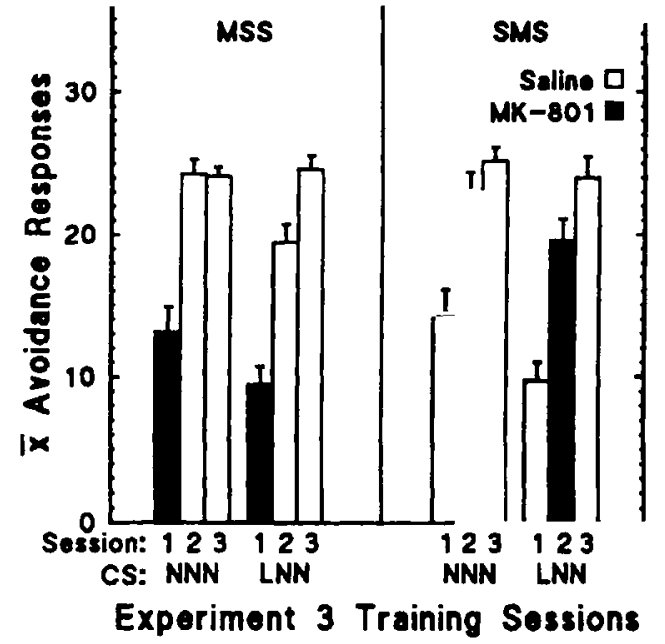

Figure 3. The mean number $( \pm S E M)$ of avoidance responses emitted by the rats in each of the four groups during each of the three training sessions. The letters identifying each drug condition indicate whether the group received $0.1-\mathrm{mg} / \mathrm{kg} \mathrm{MK-801} \mathrm{(M)}$ or saline (S) immediately after the first or second session. Two of the groups were trained with the noise (N) intensity $C S$ in all three sessions and two groups were trained with the light $(L)$ intensity $\mathrm{CS}$ in the first session and the noise $\mathrm{CS}$ in the second and third sessions.

with the noise intensity CS in the last two sessions. These groups received $\mathrm{MK}-801$ and saline in the same manner as did the first two groups.

\section{Results}

While CMT was easily detectable in this experiment, no effects of MK-801 were found. A three-factor analysis of variance for mixed designs was computed to determine the effects of CS modality (2), drug condition (2), and training session (3) on avoidance responding. This analysis revealed significant effects of modality $[F(1,24)=7.73, p<.025]$ and sessions $[F(2,48)=$ $201.51, p<.001]$ and a significant modality $\times$ sessions interaction $[F(2,48)=5.457, p<.01$; see Figure 3]. Separate analyses of the data for each session showed that rats trained with the noise intensity CS in the first session responded with significantly more avoidance responses in each of the first two sessions than did rats initially trained with the visual intensity CS, but not in the third session. Also, rats given visual training emitted significantly more avoidance responses when they first encountered the auditory CS than did the naive rats given auditory training in the first session. These findings indicated that the rats showed transfer of learning from visual to auditory training. However, no effects of MK-801 on avoidance responding could be detected in any analysis (all $F \mathrm{~s}<1.0)$.

\section{GENERAL DISCUSSION}

MK-801 appeared to have anterograde amnestic effects similar to those reported by other researchers (Caramanos \& Shapiro, 1994; Cox et al., 1994; Heale \& Har- 
ley, 1990; Parada-Turska \& Turski, 1990; G. B. Robinson, 1993; G. S. Robinson et al., 1989; Shapiro \& O'Connor, 1992; Venable \& Kelly, 1990). In Experiment 1, avoidance responding of the rats in the MSSS condition did not increase between the MK-801 session and the second session, whereas avoidance responding of the other groups reached asymptotic levels in the second session; these included the SMSS group that received MK-801 before the second session. Moreover, MK-801 did not affect avoidance responding of any other group. A variety of explanations, including impairments in processes involved in stimulus detectability (Tan, Kirk, Abraham, \& McNaughton, 1989), cue saliency (Cox et al., 1994), and attention (Dai \& Carey, 1994), have been offered to account for the effects of MK-801 on learning and memory. However, the performance of the rats in the SMSS, SSMS, and SSSM conditions in Experiment 1 did not appear to reflect disturbances in these processes. More importantly, in the first session of this experiment, MSSS rats were able to detect the auditory CS and make the association between the CS and the appropriate response well enough to perform the avoidance response at about the same level as were rats treated with saline. Although one clearly cannot rule out disturbances in these processes in this study, it seems unlikely that rats could perform the avoidance response under the influence of MK801 as well as saline rats if these processes were disrupted to the extent that no learning could occur. The same results would also argue that MK-801 did not alter the sensory or motor systems of the rats in a manner sufficient to block acquisition. The latter conclusion is also supported by the absence of effects of MK-801 on escape latencies and the generally small drug-related increases in false alarm responding in Experiments 1 and 2 which were not related to any changes in avoidance behavior. This is particularly important in view of the density of NMDA binding sites known to be located in the cortical and subcortical structures of these systems (Cotman, Monaghan, Ottersen, \& Storm-Mathisen, 1987; Monaghan \& Cotman, 1985). It seems more likely that the antagonistic effects of MK-801 on NMDA receptors may have prevented the formation, but not retrieval, of long-term memory about the auditory avoidance task rather than impair sensory or motor processes involved in cued active avoidance behavior.

Evidence of CMT from visual to auditory avoidance responding was seen in Experiments 2 and 3. The cognitive basis of CMT, often assumed to be "amodal" (Gibson, 1969), "multimodal" (Ettlinger \& Wilson, 1990), or "supramodal" (Church \& Meck, 1983), may be the result of the formation of cognitive schemas (Baddeley, 1990; Matlin, 1994) or of reference memory (Olton, Becker, \& Handelmann, 1979), both of which bridge individual modalities with stimulus information common to the modalities involved. In discrimination learning, CMT is more effective if the cues in the different modalities share a common relational feature such as relative increases in stimulus intensity (Ettlinger \& Wilson, 1990 ). For example, previous work with this avoidance task (Delay, 1986, 1988) has shown that CMT between the visual and auditory modalities is greatest when intensity cues in the two modalities are relationally the same (e.g., an increase in intensity) and is less when the intensity cues are reversed (e.g., increase in intensity in the first modality, decrease in intensity in the second). In the present study, nondrug rats trained first with a brightness CS emitted an average of 18-20 avoidance responses when they encountered the loudness CS in the second session, well above the average of $10-13$ avoidance responses emitted in the first training session by the nondrug rats in all three experiments. These findings agree well with the specific transfer effects of CMT seen in earlier studies.

Curiously, the effects of MK-801 on discrimination learning and CMT in rats appear to differ from effects reported for goldfish (Davis \& Klinger, 1994). In that study, CMT was able to prevent MK-801 from disrupting acquisition. While direct comparisons between the two species should be viewed with caution, the results of that study are clearly different from the findings of the present study. In Experiment 2, after receiving MK-801 before the second session, the rats in the SMS condition did not show the expected increase in avoidance responses from CMT. These results indicate that MK-801 disrupted processes associated with CMT which normally produce positive transfer effects during training, although the specific nature of the disturbance cannot be ascertained from these data. In addition, avoidance responding of the SMS rats did not reach the same level in the third session as that of other groups but rather was similar to the responding of the second (transfer) session of rats given saline before the first two sessions. This suggests that MK-801 also prevented new learning with the auditory CS from occurring during the session. It appears then that NMDA receptors in rats, besides being involved with the initial acquisition of a discrimination response, may also play an important role in neuronal activity participating in linking information previously learned with one sensory modality to the information available for ongoing learning with another modality. Moreover, since kinetic studies (Vezzani et al., 1989) have shown that MK-801 reaches near-peak activity 1030 min after intraperitoneal injection, the lack of MK801 effects in Experiment 3 indicates that these receptors contribute during CMT training and not to consolidation processes that may be continuing afterward.

In summary, MK-801 disrupted acquisition of a cued active avoidance response with minimum apparent disturbance of sensory or motor processes. In addition, MK-801 impaired transfer of learning from the visual modality to the auditory modality in rats, a result contrary to previous reports for goldfish. These findings suggest that NMDA receptors are an integral part of the neurological processes for transfer of learning, particularly those related to CMT. However, a number of issues remain unresolved. For example, what processes and what multimodal neural substrates which utilize NMDA receptors are involved in CMT of learning? Several cor- 
tical and subcortical structures are potential candidates, since NMDA receptors are found throughout most of the cortex, including multisensory cortices such as secondary visual areas (e.g., Oc2L cortex) which can respond to auditory as well as visual input (Toldi, Feher, \& Wolff, 1986). Another candidate is the amygdala, a structure that is often associated with aversive conditioning and that has been implicated in CMT (Murray \& Mishkin, 1985). NMDA receptors have been found in the amygdala, although not as abundantly as in the cortex (Cotman et al., 1987; Monaghan \& Cotman, 1985). And, in a somewhat broader context, are the NMDAdependent reference memory processes and neurological substrates of CMT related to the NMDA-dependent mechanisms of spatial relational or reference memory (Caramanos \& Shapiro, 1994; Shapiro \& O'Connor, 1992)?

\section{REFERENCES}

Baddeley, A. D. (1990). Human memory: Theory and practice. Boston: Allyn \& Bacon.

Caramanos, Z., \& Shapiro, M. L. (1994). Spatial memory and $N$ methyl-D-aspartate receptor antagonists APV and MK-801: Memory impairments depend on familiarity with the environment, drug dose, and training duration. Behavioral Neuroscience, 108, 30-43.

Church, R. M., \& MEck, W. H. (1983). Acquisition and cross-modal transfer of classification rules for temporal intervals. In M. L. Commons, R. J. Herrnstein, \& A. R. Wagner (Eds.), Quantitative analyses of behavior: Vol. 4. Discrimination processes (pp. 75-97). Cambridge, MA: Ballinger.

Cotman, C. W., Monaghan, D. T., Ottersen, O. P., \& StormMathisen, J. (1987). Anatomical organization of excitatory amino acid receptors and their pathways. Trends in Neurosciences, 10, 273-279.

Cox, J., Guthrie, R., Macrae, M., \& Kehoe, E. J. (1994). MK801 impairs acquisition and expression of conditioned responses in the rabbit nictitating membrane preparation. Psychobiology, 22, 156-166.

DAI, H., \& CAREY, R. J. (1994). The NMDA antagonist MK-801 can impair attention to exteroceptive stimuli. Behavioural Brain Research, 62, 149-156.

DAVIS, R. E., \& KLINGER, P. D. (1994). NMDA receptor antagonist MK-801 blocks learning of conditioned stimulus-unconditioned stimulus contiguity but not fear of conditioned stimulus in goldfish (Carassius auratus L.). Behavioral Neuroscience, 108, 935-940.

DELAY, E. R. (1986). Effects of cross-modal transfer on direct and reversal learning in the rat. Animal Learning \& Behavior, 14, 427-434.

DeLAY, E. R. (1988). Facilitative effects of cross-modality training on recovery of a conditioned avoidance response following striate cortex ablations in the rat. Neuropsychologia, 26, 661-671.

Delay, E. R., Golden, A. J., \& Steiner, N. O. (1978). A compact IC tone generator. Physiology \& Behavior, 21, 133-134.

Ettlinger, G., \& Wilson, W. A. (1990). Cross-modal performance: Behavioural processes, phylogenetic considerations and neural mechanisms. Behavioural Brain Research, 40, 169-192.

Gibson, E. J. (1969). Principles of perceptual learning and development. New York: Appleton-Century-Crofts.
Heale, V., \& HARLey, C. (1990). MK-801 and AP5 impair acquisition, but not retention, of the Morris milk maze. Pharmacology, Biochemistry \& Behavior, 36, 145-149.

HowELl, D. C. (1987). Statistical methods for psychology (2nd ed.). Boston: Duxbury.

LeE, E. H. Y., LeE, C. P., WANG, H. I., \& LiN, W. R. (1993). Hippocampal CRF, NE, and NMDA system interactions in memory processing in the rat. Synapse, 14, 144-153.

Matlin, M. W. (1994). Cognition (3rd ed.). Orlando, FL: Harcourt Brace Jovanovich.

Monaghan, D. T., \& Cotman, C. W. (1985). Distribution of $N$ methyl-D-aspartate-sensitive $\mathrm{L}-\left[{ }^{3} \mathrm{H}\right]$ glutamate-binding sites in rat brain. Journal of Neuroscience, 5, 2909-2919.

Mondadori, C., Weiskrantz, L., Buerki, H., Petschke, F., \& FagG, G. E. (1989). NMDA receptor antagonists can enhance or impair learning performance in animals. Experimental Brain Research, 75, 449-456.

Murray, E. A., \& Mishrin, M. (1985). Amygdalectomy impairs crossmodal association in monkeys. Science, 228, 604-606.

Olton, D. S., Becker, J. T., \& Handelmann, G. E. (1979). Hippocampus, space, and memory. Behavioral \& Brain Sciences, 2, 313-365.

Parada-Turska, J., \& TuRsKI, W. A. (1990). Excitatory amino acid antagonists and memory: Effect of drugs acting at $N$-methyl-Daspartate receptors in learning and memory tasks. Neuropharmacology, 29, 1111-1116.

ROBINSON, G. B. (1993). MK801 retards acquisition of a classically conditioned response without affecting conditioning-related alterations in perforant path-granule cell synaptic transmission. Psychobiology, 21, 253-264.

Robinson, G. S., JR., Crooks, G. B., JR., Shinkman, P. G., \& GallaGHER, M. (1989). Behavioral effects of MK-801 mimic deficits associated with hippocampal damage. Psychobiology, 17, 156-164.

Shapiro, M. L., \& O'ConNor, C. (1992). $N$-methyl-D-aspartate receptor antagonist MK-801 and spatial memory representation: Working memory is impaired in an unfamiliar environment but not in a familiar environment. Behavioral Neuroscience, 106, 604-612.

TAN, S., Kirk, R. C., Abraham, W. C., \& MCNaughton, N. (1989). Effects of the NMDA antagonists CPP and MK-801 on delayed conditional discrimination. Psychopharmacology, 98, 556-560.

TOLdI, J., Feher, O., \& WolfF, J. R. (1986). Sensory interactive zones in the rat cerebral cortex. Neuroscience, 18, 461-465.

Venable, N., \& Kelly, P. H. (1990). Effects of NMDA receptor antagonists on passive avoidance learning and retrieval in rats and mice. Psychopharmacology, 100, 215-221.

Vezzani, A., Serafini, R., Stasi, M. A., Caccia, S., Conti, I., Tridico, R. V., \& Samanin, R. (1989). Kinetics of MK-801 and its effect on quinolinic acid-induced seizures and neurotoxicity in rats. Journal of Pharmacology \& Experimental Therapeutics, 249, 278-283.

WeIsKRanTZ, L., \& MONDADORI, C. (1991). MK-801 can facilitate passive avoidance memory when retention is not present in control animals, and can fail to facilitate when it is present. Psychopharmacology, 105, 145-150.

WhISHAW, I. Q., \& AUER, R. N. (1989). Immediate and long-lasting effects of MK-801 on motor activity, spatial navigation in a swimming pool and EEG in the rat. Psychopharmacology, 98, 500-507.

(Manuscript received January 19, 1996; revision accepted for publication June 12, 1996.) 